Article

\title{
Survival Trends in Adults with Out-of-Hospital Cardiac Arrests after Traffic Collisions in Japan: A Population-Based Study
}

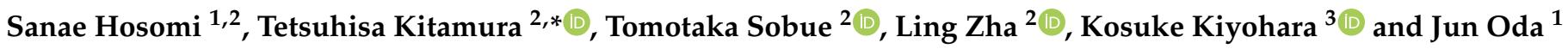 \\ 1 Department of Traumatology and Acute Critical Medicine, Graduate School of Medicine, Osaka University, \\ 2-15 Yamadaoka, Suita 565-0871, Japan; s-hosomi@hp-emerg.med.osaka-u.ac.jp (S.H.); \\ odajun@gmail.com (J.O.) \\ 2 Division of Environmental Medicine and Population Sciences, Department of Social Medicine, \\ Graduate School of Medicine, Osaka University, 2-2 Yamadaoka, Suita 565-0871, Japan; \\ tsobue@envi.med.osaka-u.ac.jp (T.S.); ivy_mist@outlook.com (L.Z.) \\ 3 Department of Food Science, Faculty of Home Economics, Otsuma Women's University, 12 Sanban-cho, \\ Chiyoda-ku, Tokyo 102-8357, Japan; kiyosuke0817@hotmail.com \\ * Correspondence: lucky_unatan@yahoo.co.jp; Tel.: +81-6-6879-3922
}

check for updates

Citation: Hosomi, S.; Kitamura, T.; Sobue, T.; Zha, L.; Kiyohara, K.; Oda, J. Survival Trends in Adults with Out-of-Hospital Cardiac Arrests after Traffic Collisions in Japan: A

Population-Based Study. J. Clin. Med. 2022, 11, 745. https://doi.org/

$10.3390 /$ jcm 11030745

Academic Editor: Alexander Oskar Spiel

Received: 20 December 2021

Accepted: 27 January 2022

Published: 29 January 2022

Publisher's Note: MDPI stays neutral with regard to jurisdictional claims in published maps and institutional affiliations.

Copyright: (C) 2022 by the authors. Licensee MDPI, Basel, Switzerland. This article is an open access article distributed under the terms and conditions of the Creative Commons Attribution (CC BY) license (https:// creativecommons.org/licenses/by/ $4.0 /)$.

\begin{abstract}
The 1-month survival rate from out-of-hospital cardiac arrest (OHCA) of cardiac origin has reportedly improved recently, at $\geq 5 \%$. However, the characteristics of patients with OHCA after a traffic collision have not been adequately evaluated in Japan. We analyzed the All-Japan Utstein Registry data of 12,577 adult patients aged $\geq 20$ years with OHCA due to traffic collisions who were resuscitated by emergency medical service personnel or bystanders and were then transported to medical institutions between 2013 and 2019. Multiple logistic regression analysis was used to assess factors potentially associated with the 1-month survival rate after OHCA. The 1-month survival rate was $1.4 \%(174 / 12,577)$. The proportion of 1 -month survival of all OHCAs after a traffic collision origin did not increase significantly (from 1.6\% [30/1919] in 2013 to 1.8\% [25/1702] in 2019), and the adjusted odds ratio for 1-year increments was 1.04 (95\% confidence interval, $0.96-1.12$ ). In multivariate analysis, men who received ventricular fibrillation, pulseless electrical activity, intravenous fluid replacement, or early emergency medical service response and had a traffic collision during daytime had significantly favorable 1-month outcomes. In Japan, the 1-month survival after OHCA of a traffic collision origin was lower than that of a cardiac origin and remains stable.
\end{abstract}

Keywords: traffic collision; mortality; out-of-hospital cardiac arrest; trauma; Japan

\section{Introduction}

Traffic collision is a major cause of hospitalization and death globally, resulting in major socioeconomic burden [1,2]. The Global Status Report on Road Safety 2018 shows that the number of deaths due to road accidents has reached 1.35 million annually [3]. The burden is disproportionately borne, with higher trends particularly observed in developing countries. However, even in developed countries, the estimated mortality rates in Europe and the United States are 11.7 and 17 per 100,000 population, respectively [3]. Despite recent advances in driver monitoring and safety assistance control systems, controversy remains as to whether patients' outcomes with traumatic out-of-hospital cardiac arrests (OHCA) following a traffic collision have improved.

Patients who die after being severely injured due to traffic collisions are usually those who undergo traumatic OHCA at the scene. Patients with traumatic cardiac arrest generally have poorer outcomes than those with non-traumatic cardiac arrest. Even with medical advancements, the ratio of survival to discharge of patients with traumatic OHCA following traffic collision tends to be lower [4-7]. A traumatic OHCA has a fundamentally different disease process, compared to that of a non-traumatic cardiac arrest, which is predominantly of primary cardiac etiology [6,7]. Thus, the prognostic factors for non-traumatic and 
traumatic cardiac arrest outcomes tend to be different, and the pathophysiology of traumatic OHCA is different due to the etiology of cardiac arrest. Therefore, other guidelines for termination of resuscitation for OHCA are applicable [8]. Improvements in the chain of survival, including developing public-access defibrillation systems and revisions to cardiopulmonary resuscitation (CPR) guidelines, have led to increased survival after OHCA of cardiac origin in some communities [4,5]; 1 -month survival was $\geq 5 \%[5,9]$. However, the epidemiological characteristics of traumatic OHCAs following traffic collisions has not been sufficiently investigated, as compared to the OHCAs of cardiac origin [10-14]. Therefore, evaluation of the characteristics, trends, and outcomes using detailed traumatic cases and understanding the factors associated with the outcomes are needed to improve survival after traumatic OHCAs following traffic collisions.

In fact, in Japan, the mortality rate associated with traffic collisions has been decreasing [15]. However, the incidence rates of traumatic OHCAs following traffic collisions in Japan, and the outcomes of traumatic OHCAs following traffic collisions are unknown. Herein, we aimed to assess nationwide trends in the incidence and outcomes of traumatic $\mathrm{OHCA}$ in adults following traffic collisions in Japan using data from the national registry.

\section{Materials and Methods}

\subsection{Study Design and Setting}

The All-Japan Utstein Registry is a prospective, population-based registry of OHCA that is based on the standardized Utstein style [16,17]. This study enrolled adult patients aged $\geq 20$ years who underwent OHCA after a traffic collision before the arrival of emergency medical services (EMS), who were resuscitated by EMS personnel or bystanders and were transported to medical institutions in Japan from 1 January 2013 to 31 December 2019. Evaluation of the detailed causes of non-cardiac OHCA did not begin until 2013. Pediatric patients with OHCA were excluded because the characteristics and outcomes of OHCA differs between children and adults [18,19].

Cardiac arrest is defined as the cessation of cardiac mechanical activity, as confirmed by the absence of signs of circulation [10]. In this registry, cardiac arrests were classified into those of presumed cardiac origin and non-cardiac origin, the latter resulting from cerebrovascular disease, asphyxia, malignant tumors, external causes, drug overuse, anaphylaxis, accidental hypothermia, traffic collision, and other causes. These diagnoses were made clinically by the physician in charge, working in collaboration with the EMS personnel. In this study, patients with traumatic OHCA following a traffic collision were included.

\subsection{EMS Organization in Japan}

Details of the EMS system in Japan have been described previously [4]. In brief, the EMS system is operated by local fire stations. When called, an ambulance is dispatched from the nearest fire station. Emergency services are provided $24 \mathrm{~h}$ every day. Most highly trained prehospital emergency care providers are called emergency life-saving technicians (ELSTs). Usually, each ambulance has a crew of three emergency providers, including at least one ELST. They are allowed to insert an intravenous line and an adjunct airway, and to use a semi-automated external defibrillator for patients with OHCA. Since July 2004, specially trained ELSTs have been permitted to perform tracheal intubation, and since April 2006, they have been permitted to administer intravenous epinephrine. Do-not-resuscitate orders or living wills are not generally accepted in Japan. EMS providers are not permitted to terminate resuscitation in the field. Therefore, almost all patients with OHCA who are treated by EMS personnel are transported to a hospital and enrolled in the All-Japan Utstein Project, excluding those with decapitation, incineration, decomposition, rigor mortis, or dependent cyanosis.

The use of an automated external defibrillator (AED) by citizens has been legally permitted since July 2004. All EMS providers perform cardiopulmonary resuscitation (CPR) according to the Japanese CPR guidelines [20]. In Japan, approximately 2 million 
citizens per year participate in community CPR programs, which include training in chest compression, mouth-to-mouth ventilation, and AED use [4,5].

\subsection{Data Collection and Quality Control}

Data were prospectively collected using a form that included data recommended in the Utstein-style reporting guidelines for cardiac arrests [16,17]. Data on patient age, sex, type of bystander witness status, first recorded cardiac rhythm, life support by EMS personnel (i.e., use of advanced life support (ALS) devices and insertion of an intravenous line), time course of resuscitation, epinephrine administration, prehospital return of spontaneous circulation (ROSC), and 1-month survival rates were obtained. Data on EMS call receipt times, arrival of the ambulance at the scene of the accident, contact with patients, initiation of CPR, defibrillation performed by EMS personnel, and arrival at the hospital were recorded using the clock of each EMS system. In cases of shock delivery by bystanders using a publicaccess AED, the patient's first recorded rhythm was regarded as ventricular fibrillation (VF) or pulseless ventricular tachycardia (VT). Information on the type of bystander CPR was obtained through observation and interviews with the bystander, which was performed by EMS personnel before leaving the scene of the accident. The data forms were completed by EMS personnel in cooperation with treating physicians. The data were integrated into the All-Japan Utstein Registry database server and were logically checked by the computer system. In cases of incomplete data forms, the Fire and Disaster Management Agency requested the provision of missing data from the respective fire stations.

All survivors who experienced OHCA were followed-up for up to 1 month after the event by the EMS personnel in charge. One-month neurological outcomes were determined by the physician responsible for treating the patient, using the cerebral performance category (CPC) scale that is measured as follows: category 1, good cerebral performance; category 2, moderate cerebral disability; category 3, severe cerebral disability; category 4 , coma or vegetative state; and category 5 , death $[16,17]$.

\subsection{Outcome Measures}

The main outcome measure was the 1-month survival rate. The secondary outcome measures included prehospital ROSC and a 1-month survival rate with neurologically favorable outcomes, defined as CPC categories 1 or $2[16,17]$.

\subsection{Statistical Analysis}

Categorical variables are presented as counts with proportions, and the $\chi^{2}$ test was used to evaluate the differences between the two groups. Continuous variables are presented as medians with interquartile ranges (IQRs), and the Wilcoxon Mann-Whitney U test was used to evaluate differences between the two groups.

The age-standardized annual incidence of OHCAs after traffic collisions was calculated by the direct method using the 2013-2019 population data from the Statistics Bureau of Japan and the 1985 Japanese model population [21,22]. Annual trends were assessed using linear trend tests. Multiple logistic regression analysis was used to assess factors associated with 1-month survival, prehospital ROSC, neurologically favorable outcomes, and adjusted odds ratios (ORs) and their 95\% confidence intervals (CIs) were calculated. As potential confounders, factors that were biologically essential and considered to be associated with clinical outcomes were included in the multivariable analyses [23]. These variables included age (20-64/65-74/ $\geq 75$ years old), sex, witness status (none/witnessed by bystanders), first documented rhythm (VF/pulseless VT/pulseless electrical activity (PEA)/asystole), bystander CPR status (any CPR/no CPR), advanced airway management (AAM) (endotracheal intubation / supraglottic airway (SGA)/none), intravascular fluid (yes/no), epinephrine (yes/no), EMS response time (call to contact with patients), contact with patients to hospital arrival, daytime (9:00 a.m.-4:59 p.m.) (yes/no), weekend/holiday (yes/no), and year of cardiac arrest. In the subgroup analysis, we conducted a multivariate 
analysis of 1-month survival from OHCAs after dividing the patients into three groups based on their age: $20-64,65-74$, and $\geq 75$ years.

All statistical analyses were conducted using STATA (version 16; StataCorp LP, College Station, TX, USA). All tests were two-tailed, and $p$-values of $<0.05$ were considered statistically significant.

\subsection{Ethics Approval}

This manuscript complies with the STROBE statement for the reporting of cohort and cross-sectional studies [24]. The study design was approved by the Ethics Committee of the Osaka University Graduate School of Medicine (approval number: 14147). The requirement for written informed consent was waived, owing to the retrospective nature of the study. Personal identifiers were not included in the Utstein records.

\section{Results}

\subsection{Eligible Patients}

Figure 1 shows an overview of the study patients based on the Utstein template. Data of a total of 866,214 adult patients with cardiac arrest were documented during these seven years. Resuscitation was attempted in 845,632 patients. After excluding 67,590 victims who were witnessed by EMS (experienced cardiac arrest after EMS arrival) and 3772 in unknown witnessed cases, 774,270 patients (280,989 bystander-witnessed arrests and 493,281 nonwitnessed arrests) were included in the analysis. Among these cases of cardiac arrest, 12,843 were due to traffic collisions. We could not obtain information on the first cardiac rhythm and bystander CPR for 266 (2.07\%) patients. Finally, the remaining 12,577 patients were eligible for our study.

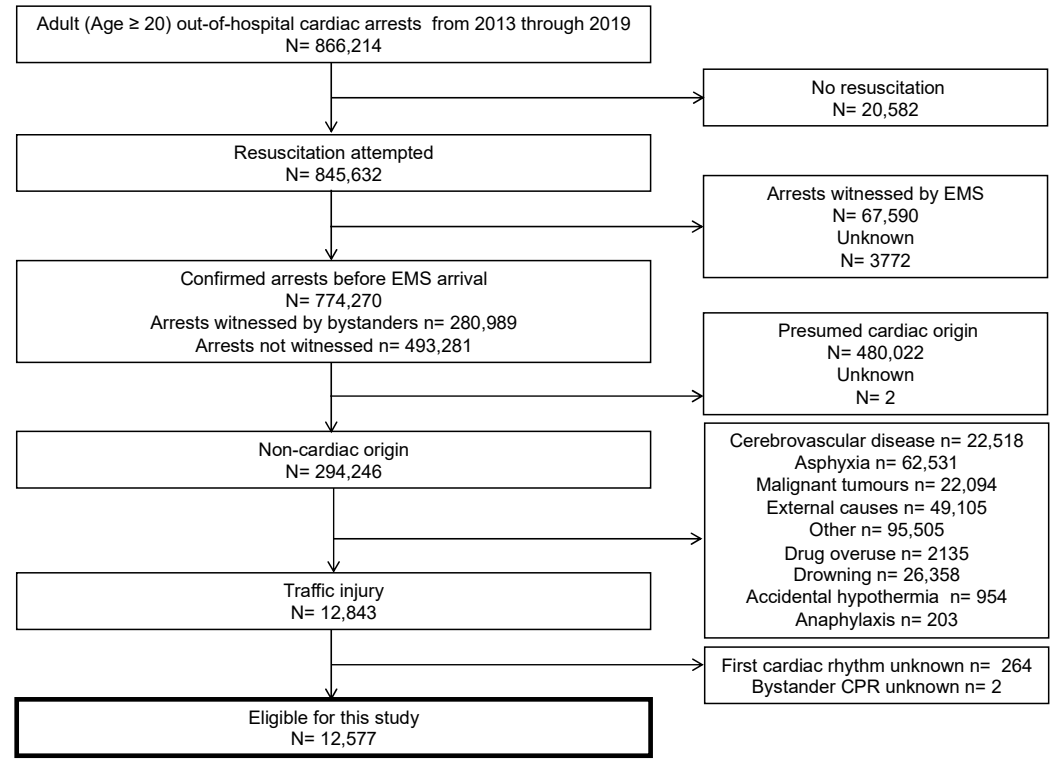

EMS = emergency medical service

Figure 1. Flow chart of the study population.

\subsection{Annual Incidence of Traumatic OHCA Following Traffic Collision}

The age-standardized annual incidence rates of traumatic OHCA following traffic collision per 100,000 persons were calculated over time (Figure 2). The incidence rate of traumatic OHCA following traffic collision did not decrease from 2013 to 2019 in all age groups except among the elderly, aged $\geq 75$ years $(p=0.148$ in 20-64 years, $p=0.052$ in $65-74$ years, and $p<0.001$ in $\geq 75$ years). 


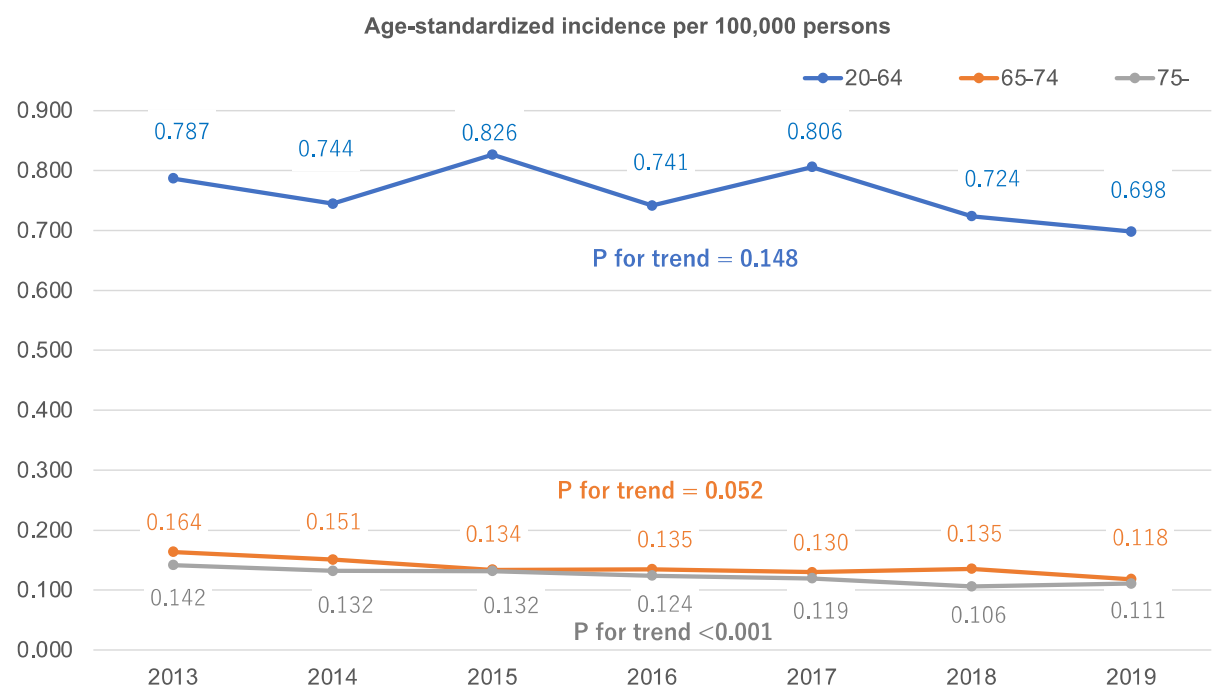

Figure 2. Age-standardized incidences of traumatic out-of-hospital cardiac arrests following traffic collisions.

\subsection{Baseline Characteristics}

The characteristics of patients and EMS personnel involved in traumatic OHCA following traffic collision are shown in Table 1, and their outcomes are shown in Table 2. The median age of all patients with traumatic OHCA following traffic collision was 65 years (range, 45-76) in 2013 and 66 years (range, 46-78) in $2019(p=0.149)$ and the proportion of men was $68.2 \%$ in 2013 and $69.5 \%$ in $2019(p=0.228)$, which were stable over time.

The number of cases with a witness $(61.9 \%$ in 2013 and $64.6 \%$ in $2019 ; p<0.001)$ significantly increased, along with the frequency of bystander CPR $(20.5 \%$ in 2013 and $27.0 \%$ in 2019; $p<0.001$ ). The first rhythm was commonly a non-shockable rhythm (pulseless electrical activity and asystole), and VF rhythm decreased from 3.3\% in 2013 to $1.0 \%$ in 2019 $(p<0.001)$. While the proportion of AAM was stable, the rate of intravascular fluid and epinephrine administration increased $(p<0.001)$. Most cases of traumatic OHCA following traffic collision occurred during nighttime and on weekends/holidays.

Table 1. Characteristics of patients and EMS involved in traumatic out-of-hospital cardiac arrests after traffic collisions.

\begin{tabular}{|c|c|c|c|c|c|c|c|c|c|}
\hline & & 2013 & 2014 & 2015 & 2016 & 2017 & 2018 & 2019 & $p$ for Trend \\
\hline & & $\mathrm{N}=1919$ & $\mathrm{~N}=1824$ & $N=1871$ & $\mathbf{N}=1775$ & $N=1839$ & $\mathrm{~N}=1702$ & $N=1647$ & \\
\hline $\begin{array}{l}\text { Age, year, median } \\
\text { (IQR) }\end{array}$ & & $\begin{array}{c}65 \\
(45-76)\end{array}$ & $\begin{array}{c}65 \\
(47-77)\end{array}$ & $\begin{array}{c}63 \\
(44-76)\end{array}$ & $\begin{array}{c}66 \\
(45-78)\end{array}$ & $\begin{array}{c}64 \\
(45-77)\end{array}$ & $\begin{array}{c}65 \\
(46-77)\end{array}$ & $\begin{array}{c}66 \\
(46-78)\end{array}$ & 0.149 \\
\hline \multirow[t]{3}{*}{ Age group, n (\%) } & $\begin{array}{l}\text { Adults aged } \\
20-64 \text { years }\end{array}$ & $\begin{array}{c}956 \\
(49.8 \%)\end{array}$ & $\begin{array}{c}891 \\
(48.8 \%)\end{array}$ & $\begin{array}{c}968 \\
(51.7 \%)\end{array}$ & $\begin{array}{c}857 \\
(48.3 \%)\end{array}$ & $\begin{array}{c}938 \\
(51.0 \%)\end{array}$ & $\begin{array}{c}839 \\
(49.3 \%)\end{array}$ & $\begin{array}{c}795 \\
(48.3 \%)\end{array}$ & 0.503 \\
\hline & $\begin{array}{l}\text { Elderly aged } \\
65-74 \text { years }\end{array}$ & $\begin{array}{c}405 \\
(21.1 \%)\end{array}$ & $\begin{array}{c}392 \\
(21.5 \%)\end{array}$ & $\begin{array}{c}355 \\
(19.0 \%)\end{array}$ & $\begin{array}{c}356 \\
(20.1 \%)\end{array}$ & $\begin{array}{c}346 \\
(18.8 \%)\end{array}$ & $\begin{array}{c}364 \\
(21.4 \%)\end{array}$ & $\begin{array}{c}315 \\
(19.1 \%)\end{array}$ & 0.190 \\
\hline & $\begin{array}{c}\text { Elderly aged } \geq \\
75 \text { years }\end{array}$ & $\begin{array}{c}558 \\
(29.1 \%)\end{array}$ & $\begin{array}{c}541 \\
(29.7 \%)\end{array}$ & $\begin{array}{c}548 \\
(29.3 \%)\end{array}$ & $\begin{array}{c}562 \\
(31.7 \%)\end{array}$ & $\begin{array}{c}555 \\
(30.2 \%)\end{array}$ & $\begin{array}{c}499 \\
(29.3 \%)\end{array}$ & $\begin{array}{c}537 \\
(32.6 \%)\end{array}$ & 0.059 \\
\hline Sex, n (\%) & Male & $\begin{array}{c}1309 \\
(68.2 \%)\end{array}$ & $\begin{array}{c}1224 \\
(67.1 \%)\end{array}$ & $\begin{array}{c}1298 \\
(69.4 \%)\end{array}$ & $\begin{array}{c}1239 \\
(69.8 \%)\end{array}$ & $\begin{array}{c}1264 \\
(68.7 \%)\end{array}$ & $\begin{array}{c}1173 \\
(68.9 \%)\end{array}$ & $\begin{array}{c}1145 \\
(69.5 \%)\end{array}$ & 0.228 \\
\hline Witness, n (\%) & $\begin{array}{l}\text { Arrests witnessed } \\
\text { by bystanders }\end{array}$ & $\begin{array}{c}1187 \\
(61.9 \%)\end{array}$ & $\begin{array}{c}1150 \\
(63.0 \%)\end{array}$ & $\begin{array}{c}1159 \\
(61.9 \%)\end{array}$ & $\begin{array}{c}1173 \\
(66.1 \%)\end{array}$ & $\begin{array}{c}1233 \\
(67.0 \%)\end{array}$ & $\begin{array}{c}1135 \\
(66.7 \%)\end{array}$ & $\begin{array}{c}1064 \\
(64.6 \%)\end{array}$ & $<0.001$ \\
\hline \multirow[t]{3}{*}{$\begin{array}{l}\text { First documented } \\
\text { rhythm, n (\%) }\end{array}$} & $\mathrm{VF} / \mathrm{pVT}$ & $63(3.3 \%)$ & $30(1.6 \%)$ & $23(1.2 \%)$ & $23(1.3 \%)$ & $31(1.7 \%)$ & $26(1.5 \%)$ & $17(1.0 \%)$ & $<0.001$ \\
\hline & PEA & $\begin{array}{c}665 \\
(34.7 \%)\end{array}$ & $\begin{array}{c}613 \\
(33.6 \%)\end{array}$ & $\begin{array}{c}619 \\
(33.1 \%)\end{array}$ & $\begin{array}{c}631 \\
(35.5 \%)\end{array}$ & $\begin{array}{c}631 \\
(34.3 \%)\end{array}$ & $\begin{array}{c}631 \\
(37.1 \%)\end{array}$ & $\begin{array}{c}601 \\
(36.5 \%)\end{array}$ & 0.023 \\
\hline & Asystole & $\begin{array}{c}1191 \\
(62.1 \%)\end{array}$ & $\begin{array}{c}1181 \\
(64.7 \%)\end{array}$ & $\begin{array}{c}1229 \\
(65.7 \%)\end{array}$ & $\begin{array}{c}1121 \\
(63.2 \%)\end{array}$ & $\begin{array}{c}1177 \\
(64.0 \%)\end{array}$ & $\begin{array}{c}1045 \\
(61.4 \%)\end{array}$ & $\begin{array}{c}1029 \\
(62.5 \%)\end{array}$ & 0.264 \\
\hline $\begin{array}{c}\text { Bystander CPR, } \\
\text { n (\%) }\end{array}$ & & $\begin{array}{c}393 \\
(20.5 \%)\end{array}$ & $\begin{array}{c}418 \\
(22.9 \%)\end{array}$ & $\begin{array}{c}442 \\
(23.6 \%)\end{array}$ & $\begin{array}{c}437 \\
(24.6 \%)\end{array}$ & $\begin{array}{c}450 \\
(24.5 \%)\end{array}$ & $\begin{array}{c}451 \\
(26.5 \%)\end{array}$ & $\begin{array}{c}444 \\
(27.0 \%)\end{array}$ & $<0.001$ \\
\hline
\end{tabular}


Table 1. Cont.

\begin{tabular}{|c|c|c|c|c|c|c|c|c|c|}
\hline & & 2013 & 2014 & 2015 & 2016 & 2017 & 2018 & 2019 & $p$ for Trend \\
\hline & & $\mathrm{N}=1919$ & $\mathbf{N}=1824$ & $N=1871$ & $\mathbf{N}=1775$ & $N=1839$ & $N=1702$ & $\mathbf{N}=1647$ & \\
\hline \multirow{3}{*}{$\begin{array}{l}\text { Advanced airway } \\
\text { management, } \mathrm{n}(\%)\end{array}$} & ETI & $57(3.0 \%)$ & $54(3.0 \%)$ & $74(4.0 \%)$ & $66(3.7 \%)$ & $74(4.0 \%)$ & $59(3.5 \%)$ & $60(3.6 \%)$ & 0.158 \\
\hline & SGA & $\begin{array}{c}514 \\
(26.8 \%)\end{array}$ & $\begin{array}{c}492 \\
(27.0 \%)\end{array}$ & $\begin{array}{c}477 \\
(25.5 \%)\end{array}$ & $\begin{array}{c}451 \\
(25.4 \%)\end{array}$ & $\begin{array}{c}443 \\
(24.1 \%)\end{array}$ & $\begin{array}{c}418 \\
(24.6 \%)\end{array}$ & $\begin{array}{c}428 \\
(26.0 \%)\end{array}$ & 0.095 \\
\hline & Non & $\begin{array}{c}1348 \\
(70.2 \%)\end{array}$ & $\begin{array}{c}1278 \\
(70.1 \%)\end{array}$ & $\begin{array}{c}1320 \\
(70.6 \%)\end{array}$ & $\begin{array}{c}1258 \\
(70.9 \%)\end{array}$ & $\begin{array}{c}1322 \\
(71.9 \%)\end{array}$ & $\begin{array}{c}1225 \\
(72.0 \%)\end{array}$ & $\begin{array}{c}1159 \\
(70.4 \%)\end{array}$ & 0.308 \\
\hline \multicolumn{2}{|l|}{$\begin{array}{l}\text { Intravascular fluid, } \\
\mathrm{n}(\%)\end{array}$} & $\begin{array}{c}440 \\
(22.9 \%)\end{array}$ & $\begin{array}{c}438 \\
(24.0 \%)\end{array}$ & $\begin{array}{c}411 \\
(22.0 \%)\end{array}$ & $\begin{array}{c}450 \\
(25.4 \%)\end{array}$ & $\begin{array}{c}505 \\
(27.5 \%)\end{array}$ & $\begin{array}{c}468 \\
(27.5 \%)\end{array}$ & $\begin{array}{c}482 \\
(29.3 \%)\end{array}$ & $<0.001$ \\
\hline \multicolumn{2}{|l|}{ Epinephrine, n (\%) } & $\begin{array}{c}245 \\
(12.8 \%)\end{array}$ & $\begin{array}{c}263 \\
(14.4 \%)\end{array}$ & $\begin{array}{c}236 \\
(12.6 \%)\end{array}$ & $\begin{array}{c}289 \\
(16.3 \%)\end{array}$ & $\begin{array}{c}325 \\
(17.7 \%)\end{array}$ & $\begin{array}{c}321 \\
(18.9 \%)\end{array}$ & $\begin{array}{c}356 \\
(21.6 \%)\end{array}$ & $<0.001$ \\
\hline \multicolumn{2}{|c|}{$\begin{array}{l}\text { Call to contact with a patient by EMS, } \\
\text { min, median (IQR) }\end{array}$} & $9(7-12)$ & $9(7-13)$ & $9(7-13)$ & $9(7-13)$ & $9(7-13)$ & $10(7-13)$ & $9(7-13)$ & $<0.001$ \\
\hline \multicolumn{2}{|c|}{$\begin{array}{c}\text { Contact to hospital arrival, min, median } \\
\text { (IQR) }\end{array}$} & $\begin{array}{c}24 \\
(17-34)\end{array}$ & $\begin{array}{c}24 \\
(17-34)\end{array}$ & $\begin{array}{c}24 \\
(17-34)\end{array}$ & $\begin{array}{c}24 \\
(18-34)\end{array}$ & $\begin{array}{c}25 \\
(18-35)\end{array}$ & $\begin{array}{c}25 \\
(18-35)\end{array}$ & $\begin{array}{c}24 \\
(18-35)\end{array}$ & 0.006 \\
\hline \multicolumn{2}{|l|}{ Daytime, n (\%) } & $\begin{array}{c}652 \\
(34.0 \%)\end{array}$ & $\begin{array}{c}629 \\
(34.5 \%)\end{array}$ & $\begin{array}{c}634 \\
(33.9 \%)\end{array}$ & $\begin{array}{c}645 \\
(36.3 \%)\end{array}$ & $\begin{array}{c}651 \\
(35.4 \%)\end{array}$ & $\begin{array}{c}594 \\
(34.9 \%)\end{array}$ & $\begin{array}{c}569 \\
(34.5 \%)\end{array}$ & 0.455 \\
\hline \multicolumn{2}{|l|}{$\begin{array}{c}\text { Weekend/Holiday, } \\
\mathrm{n}(\%)\end{array}$} & $\begin{array}{c}1409 \\
(73.4 \%)\end{array}$ & $\begin{array}{c}1281 \\
(70.2 \%)\end{array}$ & $\begin{array}{c}1345 \\
(71.9 \%)\end{array}$ & $\begin{array}{c}1243 \\
(70.0 \%)\end{array}$ & $\begin{array}{c}1347 \\
(73.2 \%)\end{array}$ & $\begin{array}{c}1243 \\
(73.0 \%)\end{array}$ & $\begin{array}{c}1165 \\
(70.7 \%)\end{array}$ & 0.830 \\
\hline
\end{tabular}

EMS, emergency medical services; ETI, endotracheal intubation; IQR, interquartile range; PEA, pulseless electrical activity; SGA, supraglottic airway; VF, ventricular fibrillation; $\mathrm{pVT}$, pulseless ventricular tachycardia.

Table 2. Annual trends in the primary and secondary outcomes of traumatic out-of-hospital cardiac arrests after traffic collisions.

\begin{tabular}{|c|c|c|c|c|c|c|c|c|c|}
\hline & Total & 2013 & 2014 & 2015 & 2016 & 2017 & 2018 & 2019 & \\
\hline & $\mathrm{N}=12,577$ & $N=1919$ & $N=1824$ & $N=1871$ & $\mathrm{~N}=1775$ & $\mathrm{~N}=1839$ & $N=1702$ & $\mathrm{~N}=1647$ & \\
\hline $\begin{array}{l}\text { One-month } \\
\text { survival, } n \\
\text { (\%) }\end{array}$ & $174(1.4 \%)$ & $30(1.6 \%)$ & $16(0.9 \%)$ & $26(1.4 \%)$ & $25(1.4 \%)$ & $22(1.2 \%)$ & $30(1.8 \%)$ & $25(1.5 \%)$ & $\begin{array}{c}\text { OR for } \\
\text { 1-year } \\
\text { increment }\end{array}$ \\
\hline $\begin{array}{c}\text { Crude OR } \\
95 \% \mathrm{CI}\end{array}$ & & reference & $\begin{array}{c}0.56 \\
(0.30-1.03)\end{array}$ & $\begin{array}{c}0.89 \\
(0.52-1.51)\end{array}$ & $\begin{array}{c}0.90 \\
(0.53-1.54)\end{array}$ & $\begin{array}{c}0.76 \\
(0.44-1.33)\end{array}$ & $\begin{array}{c}1.13 \\
(0.68-1.88)\end{array}$ & $\begin{array}{c}0.97 \\
(0.57-1.66)\end{array}$ & $\begin{array}{c}1.04 \\
(0.96-1.12)\end{array}$ \\
\hline $\begin{array}{c}\text { Adjusted OR } \\
95 \% \mathrm{CI}\end{array}$ & & reference & $\begin{array}{c}0.59 \\
(0.32-1.10)\end{array}$ & $\begin{array}{c}1.01 \\
(0.59-1.73)\end{array}$ & $\begin{array}{c}0.98 \\
(0.57-1.69)\end{array}$ & $\begin{array}{c}0.77 \\
(0.43-1.36)\end{array}$ & $\begin{array}{c}1.18 \\
(0.70-1.99)\end{array}$ & $\begin{array}{c}1.06 \\
(0.61-1.83)\end{array}$ & $\begin{array}{c}1.04 \\
(0.96-1.12)\end{array}$ \\
\hline $\begin{array}{c}\text { Prehospital } \\
\text { ROSC, n (\%) }\end{array}$ & $554(4.4 \%)$ & $92(4.8 \%)$ & $61(3.3 \%)$ & $75(4.0 \%)$ & $85(4.8 \%)$ & $76(4.1 \%)$ & $76(4.5 \%)$ & $89(5.4 \%)$ & $\begin{array}{c}\text { OR for } \\
\text { 1-year } \\
\text { increment }\end{array}$ \\
\hline $\begin{array}{c}\text { Crude OR } \\
95 \% \mathrm{CI}\end{array}$ & & reference & $\begin{array}{c}0.69 \\
(0.49-0.96)\end{array}$ & $\begin{array}{c}0.83 \\
(0.61-1.13)\end{array}$ & $\begin{array}{c}1.00 \\
(0.74-1.35)\end{array}$ & $\begin{array}{c}0.86 \\
(0.63-1.17)\end{array}$ & $\begin{array}{c}0.93 \\
(0.68-1.27)\end{array}$ & $\begin{array}{c}1.13 \\
(0.84-1.53)\end{array}$ & $\begin{array}{c}1.03 \\
(0.99-1.08)\end{array}$ \\
\hline $\begin{array}{c}\text { Adjusted OR } \\
95 \% \mathrm{CI}\end{array}$ & & reference & $\begin{array}{c}0.66 \\
(0.47-0.93)\end{array}$ & $\begin{array}{c}0.88 \\
(0.64-1.21)\end{array}$ & $\begin{array}{c}0.91 \\
(0.66-1.25)\end{array}$ & $\begin{array}{c}0.76 \\
(0.55-1.06)\end{array}$ & $\begin{array}{c}0.83 \\
(0.60-1.14)\end{array}$ & $\begin{array}{c}1.01 \\
(0.74-1.38)\end{array}$ & $\begin{array}{c}1.01 \\
(0.97-1.06)\end{array}$ \\
\hline $\begin{array}{c}\text { Neurological } \\
\text { favorable } \\
\text { outcome } \\
\text { with CPC } \\
\text { categories } 1 \\
\text { or } 2, \mathrm{n}(\%)\end{array}$ & $44(0.3 \%)$ & $7(0.4 \%)$ & $3(0.2 \%)$ & $9(0.5 \%)$ & $5(0.3 \%)$ & $2(0.1 \%)$ & $11(0.6 \%)$ & $7(0.4 \%)$ & $\begin{array}{c}\text { OR for } \\
\text { 1-year } \\
\text { increment }\end{array}$ \\
\hline $\begin{array}{c}\text { Crude OR } \\
95 \% \mathrm{CI}\end{array}$ & & reference & $\begin{array}{c}0.45 \\
(0.12-1.74)\end{array}$ & $\begin{array}{c}1.32 \\
(0.49-3.55)\end{array}$ & $\begin{array}{c}0.77 \\
(0.24-2.44)\end{array}$ & $\begin{array}{c}0.30 \\
(0.06-1.43)\end{array}$ & $\begin{array}{c}1.78 \\
(0.69-4.59)\end{array}$ & $\begin{array}{c}1.17 \\
(0.41-3.33)\end{array}$ & $\begin{array}{c}1.08 \\
(0.93-1.25)\end{array}$ \\
\hline $\begin{array}{c}\text { Adjusted OR } \\
95 \% \mathrm{CI}\end{array}$ & & reference & $\begin{array}{c}0.52 \\
(0.13-2.05)\end{array}$ & $\begin{array}{c}1.65 \\
(0.60-4.56)\end{array}$ & $\begin{array}{c}0.99 \\
(0.31-3.22)\end{array}$ & $\begin{array}{c}0.37 \\
(0.07-1.80)\end{array}$ & $\begin{array}{c}2.11 \\
(0.79-5.62)\end{array}$ & $\begin{array}{c}1.62 \\
(0.56-4.81)\end{array}$ & $\begin{array}{c}1.12 \\
(0.96-1.30)\end{array}$ \\
\hline
\end{tabular}

OR, odds ratio; $\mathrm{CI}$, confidence Interval; ROSC, return of spontaneous circulation; $\mathrm{CPC}$, cerebral performance category.

\subsection{Outcomes}

In all patients $(\mathrm{N}=12,577)$, the rates of 1-month survival, prehospital ROSC, and neurologically favorable outcomes with CPC categories 1 or 2 were $1.4 \%(n=174), 4.4 \%$ $(\mathrm{n}=554)$, and $0.3 \%(\mathrm{n}=44)$, respectively. The proportion of 1-month survival rates of traumatic OHCA following traffic collision did not significantly increase (from 1.6\% (30/1919) in 2013 to $1.5 \%(25 / 1647)$ in 2019) and the adjusted OR for annual increment was 1.04 (95\% CI, 0.96-1.12). The adjusted OR for increases in the 1-month survival rates, prehospital 
ROSC rates, and neurologically favorable outcomes after traumatic OHCA following traffic collision were almost similar to those of the 1-month survival rate (adjusted OR, 1.01; 95\% CI, 0.97-1.06; adjusted OR, 1.12; 95\% CI, 0.96-1.30; respectively).

Age-related subgroup analyses after dividing patients into three groups (20-64 years, $65-74$ years, and $\geq 75$ years) are shown in Table 3 . The 1-month survival trend of patients with traumatic OHCA following traffic collision was almost stable during the study period (20-64 years: adjusted OR, 1.11; 95\% CI, 0.99-1.24; 65-74 years: adjusted OR, 1.05; 95\% CI, $0.91-1.22$; and $\geq 75$ years: adjusted $\mathrm{OR}, 0.94 ; 95 \% \mathrm{CI}, 0.80-1.09$ ).

Table 3. One-month survival of patients with traumatic out-of-hospital cardiac arrests after traffic accidents over time by age group.

\begin{tabular}{|c|c|c|c|c|c|c|c|c|c|}
\hline & Total & 2013 & 2014 & 2015 & 2016 & 2017 & 2018 & 2019 & \\
\hline \multirow[t]{2}{*}{$20-64, n / N$} & $86 / 6244$ & $13 / 956$ & $7 / 891$ & $15 / 968$ & $10 / 857$ & $10 / 938$ & $18 / 839$ & $13 / 795$ & \\
\hline & $1.38 \%$ & $1.36 \%$ & $0.79 \%$ & $1.55 \%$ & $1.17 \%$ & $1.07 \%$ & $2.15 \%$ & $1.64 \%$ & $\begin{array}{l}\text { OR for 1-year } \\
\text { increment }\end{array}$ \\
\hline $\begin{array}{c}\text { Crude OR } \\
95 \% \mathrm{CI}\end{array}$ & & reference & $\begin{array}{c}0.57 \\
(0.23-1.45)\end{array}$ & $\begin{array}{c}1.14 \\
(0.54-2.41)\end{array}$ & $\begin{array}{c}0.86 \\
(0.37-1.96)\end{array}$ & $\begin{array}{c}0.78 \\
(0.34-1.79)\end{array}$ & $\begin{array}{c}1.59 \\
(0.77-3.27)\end{array}$ & $\begin{array}{c}1.21 \\
(0.56-2.62)\end{array}$ & $\begin{array}{c}1.08 \\
(0.97-1.20)\end{array}$ \\
\hline $\begin{array}{c}\text { Adjusted OR } \\
95 \% C I\end{array}$ & & reference & $\begin{array}{c}0.75 \\
(0.29-1.94)\end{array}$ & $\begin{array}{c}1.50 \\
(0.69-3.26)\end{array}$ & $\begin{array}{c}1.10 \\
(0.47-2.60)\end{array}$ & $\begin{array}{c}0.96 \\
(0.41-2.27)\end{array}$ & $\begin{array}{c}1.87 \\
(0.88-3.97)\end{array}$ & $\begin{array}{c}1.67 \\
(0.74-3.75)\end{array}$ & $\begin{array}{c}1.11 \\
(0.99-1.24)\end{array}$ \\
\hline \multirow[t]{2}{*}{$65-74, \mathrm{n} / \mathrm{N}$} & $47 / 2533$ & $6 / 405$ & $7 / 392$ & $7 / 355$ & $5 / 356$ & $8 / 346$ & $7 / 364$ & $7 / 315$ & \\
\hline & $1.86 \%$ & $1.48 \%$ & $1.79 \%$ & $1.97 \%$ & $1.40 \%$ & $2.31 \%$ & $1.92 \%$ & $2.22 \%$ & $\begin{array}{l}\text { OR for 1-year } \\
\text { increment }\end{array}$ \\
\hline $\begin{array}{c}\text { Crude OR } \\
95 \% \mathrm{CI}\end{array}$ & & reference & $\begin{array}{c}1.21 \\
(0.40-3.63)\end{array}$ & $\begin{array}{c}1.34 \\
(0.45-4.02)\end{array}$ & $\begin{array}{c}0.95 \\
(0.29-3.13)\end{array}$ & $\begin{array}{c}1.57 \\
(0.54-4.58)\end{array}$ & $\begin{array}{c}1.30 \\
(0.43-3.92)\end{array}$ & $\begin{array}{c}1.51 \\
(0.50-4.54)\end{array}$ & $\begin{array}{c}1.06 \\
(0.91-1.22)\end{array}$ \\
\hline $\begin{array}{c}\text { Adjusted OR } \\
95 \% \mathrm{CI}\end{array}$ & & reference & $\begin{array}{c}1.08 \\
(0.35-3.31)\end{array}$ & $\begin{array}{c}1.53 \\
(0.50-4.69)\end{array}$ & $\begin{array}{c}1.00 \\
(0.30-3.40)\end{array}$ & $\begin{array}{c}1.30 \\
(0.42-4.00)\end{array}$ & $\begin{array}{c}1.37 \\
(0.44-4.24)\end{array}$ & $\begin{array}{c}1.47 \\
(0.48-4.53)\end{array}$ & $\begin{array}{c}1.05 \\
(0.91-1.22)\end{array}$ \\
\hline \multirow[t]{2}{*}{$\geq 75, \mathrm{n} / \mathrm{N}$} & $41 / 3800$ & $11 / 558$ & $2 / 541$ & $4 / 548$ & $10 / 562$ & $4 / 555$ & $5 / 499$ & $5 / 537$ & \\
\hline & $1.08 \%$ & $1.97 \%$ & $0.37 \%$ & $0.73 \%$ & $1.78 \%$ & $0.72 \%$ & $1.00 \%$ & $0.93 \%$ & $\begin{array}{l}\text { OR for 1-year } \\
\text { increment }\end{array}$ \\
\hline $\begin{array}{c}\text { Crude OR } \\
95 \% \text { CI }\end{array}$ & & reference & $\begin{array}{c}0.18 \\
(0.04-0.84)\end{array}$ & $\begin{array}{c}0.37 \\
(0.12-1.16)\end{array}$ & $\begin{array}{c}0.90 \\
(0.38-2.14)\end{array}$ & $\begin{array}{c}0.36 \\
(0.11-1.14)\end{array}$ & $\begin{array}{c}0.50 \\
(0.17-1.46)\end{array}$ & $\begin{array}{c}0.47 \\
(0.16-1.35)\end{array}$ & $\begin{array}{c}0.94 \\
(0.80-1.09)\end{array}$ \\
\hline $\begin{array}{c}\text { Adjusted OR } \\
95 \% \mathrm{CI}\end{array}$ & & reference & $\begin{array}{c}0.18 \\
(0.04-0.81)\end{array}$ & $\begin{array}{c}0.37 \\
(0.12-1.19)\end{array}$ & $\begin{array}{c}0.81 \\
(0.33-1.95)\end{array}$ & $\begin{array}{c}0.38 \\
(0.12-1.23)\end{array}$ & $\begin{array}{c}0.51 \\
(0.17-1.51)\end{array}$ & $\begin{array}{c}0.44 \\
(0.15-1.28)\end{array}$ & $\begin{array}{c}0.94 \\
(0.80-1.09)\end{array}$ \\
\hline
\end{tabular}

OR, odds ratio; $\mathrm{CI}$, confidence interval.

\subsection{Factors Related to Mortality}

Table 4 shows the factors contributing to 1-month survival after traumatic OHCA following a traffic collision. With regard to 1-month survival, male sex (adjusted OR, 1.56 ; 95\% CI, 1.08-2.25), VF as the first documented rhythm (adjusted OR, 15.32; 95\% CI, 8.08-29.07), PEA as the first documented rhythm (adjusted OR, 5.77; 95\% CI, 3.92-8.49), intravenous fluid levels (adjusted OR, 3.00; 95\% CI, 1.99-4.50), early EMS response time (adjusted OR for 1-min-increment 0.96; 95\% CI, 0.93 to 1.00), and daytime (adjusted OR, $1.89 ; 95 \%$ CI, 1.39-2.58) were associated with favorable outcomes. However, AAM by SGA (adjusted OR, 0.50; 95\% CI, 0.33-0.76) and epinephrine (adjusted OR, 0.42; 95\% CI, $0.25-0.69$ ) were not associated with favorable outcomes.

Table 4. Factors associated with primary outcomes.

\begin{tabular}{ccccccccc}
\hline & All (N) & $\begin{array}{c}\text { 1 Month } \\
\text { Survival (N) }\end{array}$ & $\mathbf{( \% )}$ & Crude OR & 95\% CI & $\begin{array}{c}\text { Adjusted } \\
\text { OR }\end{array}$ & 95\% CI \\
\hline Age group, n (\%) & $\begin{array}{c}\text { Adults aged 18-64 } \\
\text { years }\end{array}$ & 6244 & 86 & $1.38 \%$ & (reference) & & (reference) & \\
& $\begin{array}{c}\text { Elderly aged 65-74 } \\
\text { years }\end{array}$ & 2533 & 47 & $1.86 \%$ & 1.35 & $(0.95-1.94)$ & 1.26 & $(0.87-1.83)$ \\
& $\begin{array}{c}\text { Elderly aged } \geq 75 \\
\text { years }\end{array}$ & 3800 & 41 & $1.08 \%$ & 0.78 & $(0.54-1.14)$ & 0.70 & $(0.47-1.03)$ \\
Sex, n (\%) & $\begin{array}{c}\text { Female } \\
\text { Male }\end{array}$ & 3925 & 42 & $1.07 \%$ & $\begin{array}{c}\text { (reference) } \\
\text { (reference) } \\
1.43\end{array}$ & $(1.01-2.03)$ & $(1.08-2.25)$ \\
\hline
\end{tabular}


Table 4. Cont.

\begin{tabular}{|c|c|c|c|c|c|c|c|c|}
\hline & & All (N) & $\begin{array}{c}1 \text { Month } \\
\text { Survival (N) }\end{array}$ & $(\%)$ & Crude OR & $95 \%$ CI & $\begin{array}{l}\text { Adjusted } \\
\text { OR }\end{array}$ & $95 \%$ CI \\
\hline \multirow[t]{2}{*}{ Witness, n (\%) } & $\begin{array}{l}\text { Arrests witnessed by } \\
\text { bystanders }\end{array}$ & 8101 & 117 & $1.44 \%$ & 1.14 & $(0.83-1.56)$ & 1.01 & $(0.73-1.42)$ \\
\hline & Arrests not witnessed & 4476 & 57 & $1.27 \%$ & (reference) & & (reference) & \\
\hline \multirow{2}{*}{$\begin{array}{c}\text { First } \\
\text { documented } \\
\text { rhythm, n (\%) }\end{array}$} & $\mathrm{VF} / \mathrm{pVT}$ & 213 & 15 & $7.04 \%$ & 16.25 & $(8.77-30.09)$ & 15.32 & $(8.08-29.07)$ \\
\hline & $\begin{array}{c}\text { PEA } \\
\text { Asystole }\end{array}$ & $\begin{array}{l}4391 \\
7973\end{array}$ & $\begin{array}{c}122 \\
37\end{array}$ & $\begin{array}{l}2.78 \% \\
0.46 \%\end{array}$ & $\begin{array}{c}6.13 \\
\text { (reference) }\end{array}$ & $(4.24-8.87)$ & $\begin{array}{c}5.77 \\
\text { (reference) }\end{array}$ & $(3.92-8.49)$ \\
\hline \multirow{2}{*}{$\begin{array}{l}\text { Bystander CPR, } \\
\text { n (\%) }\end{array}$} & No & 9542 & 138 & $1.45 \%$ & (reference) & & (reference) & \\
\hline & Yes & 3035 & 36 & $1.19 \%$ & 0.82 & $(0.57-1.18)$ & 0.70 & $(0.47-1.02)$ \\
\hline \multirow{2}{*}{$\begin{array}{c}\text { Advanced } \\
\text { airway } \\
\text { management, } \mathrm{n} \\
(\%)\end{array}$} & ETI & 444 & 9 & $2.03 \%$ & 1.34 & $(0.68-2.66)$ & 1.06 & $(0.50-2.23)$ \\
\hline & $\begin{array}{l}\text { SGA } \\
\text { None }\end{array}$ & $\begin{array}{l}3223 \\
8910\end{array}$ & $\begin{array}{c}30 \\
135\end{array}$ & $\begin{array}{l}0.93 \% \\
1.52 \%\end{array}$ & $\begin{array}{c}0.61 \\
\text { (reference) }\end{array}$ & $(0.41-0.91)$ & $\begin{array}{l}0.50 \\
\text { (reference) }\end{array}$ & $(0.33-0.76)$ \\
\hline \multirow[t]{2}{*}{$\begin{array}{l}\text { Intravascular } \\
\text { fluid, } \mathrm{n}(\%)\end{array}$} & No & 9383 & 111 & $1.18 \%$ & (reference) & & (reference) & \\
\hline & Yes & 3194 & 63 & $1.97 \%$ & 1.68 & $(1.23-2.30)$ & 3.00 & $(1.99-4.50)$ \\
\hline \multirow[t]{2}{*}{$\begin{array}{l}\text { Epinephrine, } \mathrm{n} \\
(\%)\end{array}$} & No & 10,542 & 144 & $1.37 \%$ & (reference) & & (reference) & \\
\hline & Yes & 2035 & 30 & $1.47 \%$ & 1.08 & $(0.73-1.61)$ & 0.42 & $(0.25-0.69)$ \\
\hline \multicolumn{2}{|c|}{$\begin{array}{l}\text { Call to contact with a patient by EMS, min, } \\
\text { median (IQR) }\end{array}$} & & & & 0.93 & $(0.89-0.96)$ & 0.96 & $(0.93-1.00)$ \\
\hline \multicolumn{3}{|c|}{$\begin{array}{c}\text { Contact to hospital arrival, min, median } \\
\text { (IQR) }\end{array}$} & & & 0.99 & $(0.98-1.00)$ & 1.00 & $(0.99-1.01)$ \\
\hline \multirow[t]{2}{*}{ Daytime, n (\%) } & No & 8203 & 91 & $1.11 \%$ & (reference) & & (reference) & \\
\hline & Yes & 4374 & 83 & $1.90 \%$ & 1.72 & $(1.28-2.33)$ & 1.89 & $(1.39-2.58)$ \\
\hline \multirow[t]{2}{*}{$\begin{array}{l}\text { Weekend/Holiday, } \\
\text { n (\%) }\end{array}$} & No & 11,992 & 168 & $1.40 \%$ & (reference) & & (reference) & \\
\hline & Yes & 585 & 6 & $1.03 \%$ & 0.73 & $(0.32-1.65)$ & 0.60 & $(0.24-1.47)$ \\
\hline
\end{tabular}

PEA, pulseless electrical activity; SGA, supraglottic airway; ETI, endotracheal intubation; IQR, interquartile range $\mathrm{VF}$, ventricular fibrillation; OR, odds ratio; CI, confidence interval; CPR, cardiopulmonary resuscitation; EMS, emergency medical services.

\section{Discussion}

This study described the actual situation of the incidence and outcomes of traumatic OHCAs in adults following traffic collisions, thereby providing valuable information to appropriately manage patients and improve survival. This study, based on the extensive OHCA registry of Japan, showed that the 1-month survival after a traumatic OHCA following traffic collision was $1.4 \%$, and that the survival trends did not improve year-by-year. To further improve survival after OHCA, attention should be paid to the epidemiological characteristics of traumatic OHCA following a traffic collision, as is paid to OHCAs of cardiac origin.

More than 120,000 Japanese individuals experience OHCA annually [4,5]. Although the survival rate of OHCA is improving, $\geq 5 \%$ of individuals survive 1 month after experiencing OHCA $[4,5,9,25]$. We found that patients with traumatic OHCA have an extremely low survival rate, possibly due to the changing epidemiology of patients with traumatic OHCA following traffic collisions in older patients with a higher number of comorbidities. Globally, Japan is now one of the few countries with a super-aged population. However, the annual incidence rates of the elderly, aged $\geq 75$ years, with traumatic OHCA following traffic collisions decreased during the study period.

Survival after traumatic OHCA is rare, even with maximal resuscitative efforts. Most importantly, efforts should focus on the prevention of traumatic OHCA following traffic collisions because most cases are preventable [1-4]. To reduce trauma-related deaths caused by motor vehicle collisions (MVCs), the Japanese Road Traffic Act was revised in June 2002, and it imposes severe fines for traffic offenses. For example, the incidence of fatal collisions caused by drunk drivers has decreased since then. It was reported that the 
age-standardized incidences per 100,000 persons ranged from 1.6 in 2005 to 1.4 in 2011 $(p=0.229)$ and the unadjusted 1-month survival rate ranged from $0.9 \%(1 / 116)$ in 2005 to $2.6 \%(3 / 115)$ in 2011 ( $p=0.027)$ in Osaka, Japan [21]. However, in our study, this rate seems to be plateauing in our study, thereby warranting further improvement. There are three approaches to road safety: the traditional approach, which focuses on human errors and road users; the systemic approach, which includes sustainable safety and safe systems; and the vision zero, which is a multi-national road traffic safety project that aims to achieve a highway system with no fatalities or serious injuries following traffic collision. More recently, traffic collisions caused by older individuals have become a serious social problem, and a cognitive test for the license renewal procedure is required for drivers aged $\geq 75$ years to reduce MVCs in Japan. The test has been obligatory since June 2009 [26], helping more elderly people to voluntarily return their license. Furthermore, many automobile manufacturers strive to improve safe driving support systems and devices, such as improved occupant recognition performance of the driver monitoring system, vehicle motion control technology, and the introduction of "connected safety" technologies, such as the Advanced Automatic Collision Notification and infrastructure coordination.

Our study showed the proportion of bystander CPR for traumatic OHCA cases following a traffic collision in Japan might be lower than that of OHCAs of cardiac origin [4]. There might be differences in the proficiency of bystander basic life support (BLS) procedures between cardiac and traumatic OHCA cases. Some traumatic deaths following MVCs occur due to severe brain injury or hemorrhage shock [27]. In serious trauma cases, wherein the patients experience a cardiac arrest at the scene, the effectiveness of BLS might be clinically limited. Furthermore, EMS response time was an independent predictor of favorable outcomes in the multivariate analysis. As recommended in the CPR guidelines [19,28], activating EMS plays a key role in the "chain of survival".

In Japan, EMS personnel are not allowed to perform certain advanced interventions (for example, surgical airway, chest drain, or intraosseous access). They are not allowed to insert chest tubes, even when pneumothorax is suspected. This means that EMS personnel in Japan are permitted to perform only intravenous catheter insertion and fluid infusion, intravenous epinephrine administration, and endotracheal intubation. EMS personnel need permission or instructions from a medical director in each municipality to perform ALS on a case-to-case basis, which may prevent EMS personnel from providing timely treatment. In this study, very few patients received prehospital ALS procedures (i.e., intravenous catheter insertion, epinephrine administration, or AAM by SGA). However, in our study, intravenous access was associated with a reduction in hospital mortality among patients. This may be because traumatic OHCA is mostly due to blood loss, pneumothorax, or pericardial tamponade [27]. The etiology of cardiac arrest among the patients included in this study was blunt trauma [29]. Conversely, the multivariate analysis also showed that epinephrine and AAM by SGA were not effective. Indeed, they seemed to be associated with worse outcome, as suggested by previous observational studies [30,31]. However, we considered that there could be an inversion phenomenon of cause and effect; it would be difficult to assess the effect of ALS measures in this observational study because the EMS personnel in Japan could provide ALS measures only for OHCA patients who did not respond to BLS. Recently, some studies suggested that earlier epinephrine administration and endotracheal intubation contributed to improving outcomes after OHCA [25]. These earlier interventions would be surrogate parameters for a team that works faster and/or is more experienced overall. Further investigations by other cohorts and randomized controlled trials are needed to confirm these associations. Another effective option may be to permit EMS personnel to perform ALS based on their own judgment and/or expand the range of available procedures that EMS personnel can learn, practice, and perform. In addition, verifying these effects on prehospital emergency care and in-hospital treatment is essential [32].

Recent reports investigated the association between the time and day of the week with outcomes from adult OHCAs [33,34] and in-hospital cardiac arrests [35]. The survival 
rates of those who experienced cardiac arrests during nights and on weekends was found to be lower than those who experienced them during the day and on weekdays. This study demonstrated that traumatic OHCA following traffic collisions occurs more often at nighttime than during daytime and more often on weekends/holidays than on weekdays. Furthermore, the 1-month survival rate of traumatic OHCA cases following traffic collisions was significantly lower during nights than during days. This poorer outcome might be due to a longer reporting time of the accident or due to understaffed hospitals during the night. In addition, factors such as male sex were also independent predictors of better outcomes after traumatic OHCAs following traffic collision in a multivariate analysis. Our study is consistent with the findings of previous studies, although the reason is unknown [36].

This study has some inherent limitations. Our Utstein-based data did not include data on in-hospital treatments, such as trauma care (fluid resuscitation, emergent thoracotomy, and aortic cross-clamping) [27], post-cardiac arrest care [37], and hospital staffing. Furthermore, the autopsy data were not obtained because our data were collected in accordance with the Utstein-style guidelines by EMS personnel. Therefore, our study could not identify the detailed origin of the presumed OHCAs nor estimate OHCAs due to a medical origin (e.g., pulmonary embolism or cardiac arrhythmias, such as VF/pVT) in those caused by traffic injuries. Second, our results might not be fully applicable to other countries, including the United States and Europe, which have different EMS and medical systems. Currently, procedures by paramedics on the scene are limited in Japan; there is a need for them to be allowed to perform other procedures (e.g., insertion of chest tubes) to reduce the mortality rates of OHCA due to traffic collision. Therefore, further investigations of other cohorts are needed to confirm these associations and to address their generalizability. Third, there might be unmeasured confounding factors that might have influenced the association between traumatic OHCA following traffic collisions and outcomes. In addition, we could not identify the most severely injured organ from the registry. We did not know whether the victims were pedestrians, cyclists, motorcyclists, or drivers/passengers. This study does not include the COVID-19 pandemic period, and the results may change if a longer observation period is considered [38]. Finally, as with all epidemiological studies, data integrity, validity, and ascertainment bias are potential limitations. The use of uniform data collection based on Utstein-style guidelines for reporting cardiac arrest, a large sample size, and a population-based design to cover all known OHCAs in Japan was intended to minimize these potential sources of bias.

\section{Conclusions}

The large OHCA registry of Japan indicated that the 1-month survival rate after traumatic OHCAs following traffic collisions is lower than those of cardiac origin. Survival trends did not improve with year, and survival did not differ by age. The prevention of traumatic OHCAs and a greater range of prehospital treatment methods for traumatic $\mathrm{OHCAs}$ following traffic collision are warranted to improve survival.

Author Contributions: S.H., T.S. and T.K. designed the study and wrote the manuscript. S.H., L.Z., K.K. and T.K. performed the statistical analyses. J.O. critically revised the manuscript for important intellectual content. All authors have read and agreed to the published version of the manuscript.

Funding: This work was supported by a Grant-in-Aid for Scientific Research (C) from the Japan Society for the Promotion of Science (18K08886 and 21K09016) to S.H. This study was supported by a scientific research grant from the Ministry of Education, Culture, Sports, Science, and Technology of Japan (19K09393) to T.K.

Institutional Review Board Statement: This study was approved by the ethics committee of the Osaka University Graduate School of Medicine (No. 14147).

Informed Consent Statement: Personal identifiers were removed beforehand from the All-Japan Utstein Registry; thus, the need for obtaining informed consent was waived. 
Data Availability Statement: The data that support the findings of this study are available from the All-Japan Utstein Registry; restrictions apply to the availability of these data, which were used under license for the current study, and are therefore, not publicly available. Data are, however, available from the authors upon reasonable request and with permission from the All-Japan Utstein Registry.

Acknowledgments: The authors thank all the EMS personnel and physicians in Japan, the Fire and Disaster Management Agency, and the Institute for Fire Safety and Disaster Preparedness of Japan for their generous cooperation in establishing and maintaining the Utstein database. We thank our colleagues from the Osaka University Center of Medical Data Science and Advanced Clinical Epidemiology Investigator's Research Project for providing insight to and expertise for our research.

Conflicts of Interest: No competing financial interest exist.

\section{References}

1. Ameratunga, S.; Hijar, M.; Norton, R. Road-traffic injuries: Confronting disparities to address a global-health problem. Lancet 2006, 367, 1533-1540. [CrossRef]

2. Dan Chisholm, H.N. Road Traffic Injury Prevention: An Assessment of Risk Exposure and Intervention Cost-Effectiveness in Different World Regions; Contract; Department of Health Systems Financing: Geneva, Switzerland, 2008.

3. World Health Organization. WHO Global Status Report on Road Safety 2013: Supporting a Decade of Action; World Health Organization: Geneva, Switzerland, 2013; Available online: https://apps.who.int/iris/handle/10665/78256 (accessed on 23 November 2021).

4. Kitamura, T.; Iwami, T.; Kawamura, T.; Nagao, K.; Tanaka, H.; Hiraide, A. Nationwide public-access defibrillation in Japan. N. Engl. J. Med. 2010, 362, 994-1004. [CrossRef] [PubMed]

5. Fire and Disaster Management Agency of Japan. Effect of First Aid for Cardiopulmonary Arrest; FDMA Ambulance Service Planning Office: Tokyo, Japan, 2021. Available online: https://www.fdma.go.jp/publication/rescue/post-2.html (accessed on 23 November 2021).

6. Evans, C.C.; Petersen, A.; Meier, E.N.; Buick, J.E.; Schreiber, M.; Kannas, D.; Austin, M.A. Prehospital traumatic cardiac arrest: Management and outcomes from the resus-citation outcomes consortium epistry-trauma and PROPHET registries. J. Trauma Acute Care Surg. 2016, 81, 285-293. [CrossRef] [PubMed]

7. Gräsner, J.T.; Wnent, J.; Seewald, S.; Meybohm, P.; Fischer, M.; Paffrath, T.; Wafaisade, A.; Bein, B.; Rolf Lefering \& German Resuscitation Registry Working Group, Trauma Registry of the German Society for Trauma Surgery (DGU). Cardiopulmonary resuscitation traumatic cardiac arrest-there are survivors. An analysis of two national emergency registries. Crit. Care 2011, 15, R276. [CrossRef] [PubMed]

8. Hopson, L.R.; Hirsh, E.; Delgado, J.; Domeier, R.M.; McSwain, N.E.; Krohmer, J. Guidelines for withholding or termination of resuscitation in prehospital traumatic cardiopulmonary arrest: Joint position statement of the National Association of EMS Physicians and the American College of Surgeons Committee on Trauma. J. Am. Coll. Surg. 2003, 196, 106-112. [CrossRef]

9. Fukuda, T.; Ohashi-Fukuda, N.; Matsubara, T.; Doi, K.; Kitsuta, Y.; Nakajima, S.; Yahagi, N. Trends in outcomes for out-of-hospital cardiac arrest by age in Japan: An observational study. Medicine 2015, 94, e2049. [CrossRef]

10. Kitamura, T.; Iwami, T.; Kawamura, T.; Nagao, K.; Tanaka, H.; Hiraide, A. Bystander-initiated rescue breathing for out-of-hospital cardiac arrests of non-cardiac origin. Circulation 2010, 122, 293-299. [CrossRef]

11. Kuisma, M.; Alaspää, A. Out-of-hospital cardiac arrests of non-cardiac origin. Epidemiology and outcome. Eur. Heart J. 1997, 18, 1122-1128. [CrossRef] [PubMed]

12. Engdahl, J.; Bang, A.; Karlson, B.W.; Lindqvist, J.; Herlitz, J. Characteristics and outcome among patients suffering from out of hospital cardiac arrest of non-cardiac aetiology. Resuscitation 2003, 48, 33-41. [CrossRef]

13. Hess, E.P.; Campbell, R.L.; White, R.D. Epidemiology, trends, and outcome of out-of-hospital cardiac arrest of non-cardiac origin. Resuscitation 2007, 72, 200-207. [CrossRef]

14. Engdahl, J.; Holmberg, M.; Karlson, B.W.; Luepker, R.; Herlitz, J. The epidemiology of out-of-hospital 'sudden' cardiac arrest. Resuscitation 2002, 52, 235-245. [CrossRef]

15. E-Stat Portal Site of Official Statistics of Japan. Available online: https://www.stat.go.jp/data/jinsui/2013np/ (accessed on 23 November 2021).

16. Cummins, R.O.; Chamberlain, D.A.; Abramson, N.S.; Allen, M.; Baskett, P.J.; Becker, L.; Bossaert, L.; Delooz, H.H.; Dick, W.F.; Eisenberg, M.S. Recommended guideline for uniform reporting of data from out-of-hospital cardiac arrest: The Utstein style: A statement for health professionals from a task force of the American Heart Association, the European Resuscitation Council, the Heart and Stroke Foundation of Canada, and the Australian Resuscitation Council. Circulation 1991, 84, 960-975. [PubMed]

17. Jacobs, I.; Nadkarni, V.; Bahr, J.; Berg, R.A.; Billi, J.E.; Bossaert, L.; Cassan, P.; Coovadia, A.; D'Este, K.; Finn, J.; et al. Cardiac arrest and cardiopulmonary resuscitation outcome reports: Update and sim-plification of the Utstein templates for resuscitation registries: A statement for healthcare professionals from a task force of the International Liaison Committee on Resuscitation (American Heart Association, European Resuscitation Council, Australian Resuscitation Council, New Zealand Resuscitation Council, Heart and Stroke Foundation of Canada, Inter American Heart Foundation, Resuscitation Councils of Southern Africa). Circulation 2004, 110, 3385-3397. [PubMed] 
18. Nitta, M.; Iwami, T.; Kitamura, T.; Nadkarni, V.M.; Berg, R.A.; Shimizu, N.; Ohta, K.; Nishiuchi, T.; Hayashi, Y.; Hiraide, A.; et al. Age-specific differences in outcomes after out-of-hospital cardiac arrests. Pediatrics 2011, 128, e812-e820. [CrossRef]

19. Atkins, D.L.; Everson-Stewart, S.; Sears, G.K.; Daya, M.; Osmond, M.H.; Warden, C.R.; Berg, R.A.; The Resuscitation Outcomes Consortium Investigators. Epidemiology and outcomes from out-of-hospital cardiac arrest in children: The Resuscitation Outcomes Consortium Epistry-Cardiac Arrest. Circulation 2009, 119, 1484-1491. [CrossRef]

20. Japan Resuscitation Council. 2010 Japanese Guidelines for Emergency Care and Cardiopulmonary Resuscitation, 1st ed.; Health Shuppansha: Tokyo, Japan, 2011. (In Japanese)

21. 2013 Population Data by Statistics Bureau of Japan. Available online: https://www.e-stat.go.jp/stat-search/files?page=1\& layout $=$ datalist $\&$ toukei $=00200524 \&$ tstat $=000000090001 \&$ cycle $=7 \& y e a r=20130 \&$ month $=0 \&$ tclass $1=000001011679$ (accessed on 15 December 2021). (In Japanese)

22. Ministry of Health, Labour and Welfare. Vital Statistics of Japan 2005; Health and Welfare Statistics Association: Tokyo, Japan, 2007.

23. Kitamura, T.; Kiyohara, K.; Sakai, T.; Iwami, T.; Nishiyama, C.; Kajino, K.; Nishiuchi, T.; Hayashi, Y.; Katayama, Y.; Yoshiya, K.; et al. Epidemiology and outcome of adult out-of-hospital cardiac arrest of non-cardiac origin in Osaka: A population-based study. BMJ Open 2014, 4, e006462. [CrossRef]

24. Gallo, V.; Egger, M.; McCormack, V.; Farmer, P.B.; Ioannidis, J.P.; Kirsch-Volders, M.; Matullo, G.; Phillips, D.H.; Schoket, B.; Stromberg, U.; et al. Ulf Stromberg Strengthening the reporting of observational studies in epidemiology-molecular epidemiology STROBE-ME: An extension of the STROBE statement. J. Clin. Epidemiol. 2011, 64, 1350-1363. [CrossRef]

25. Nakahara, S.; Tomio, J.; Ichikawa, M.; Nakamura, F.; Nishida, M.; Takahashi, H.; Morimura, N.; Sakamoto, T. Association of bystander interventions with neurologically intact survival among patients with bystander-witnessed out-of-hospital cardiac arrest in Japan. JAMA 2015, 314, 247-254. [CrossRef]

26. Japanese Traffic Bureau, HP. Available online: https://www.npa.go.jp/english/bureau/traffic/index.html (accessed on 23 November 2021).

27. Feliciano, D.V.; Mattox, K.L.; Moore, E.E. (Eds.) Trauma, 9th ed.; McGraw-Hill Education: New York, NY, USA, 2021.

28. International Liaison Committee on Resuscitation. 2010 International consensus on cardiopulmonary resuscitation and emergency cardiovascular care science with treatment recommendations. Circulation 2010, 122, S283-S290. [CrossRef]

29. Katayama, Y.; Kitamura, T.; Kiyohara, K.; Ishida, K.; Hirose, T.; Nakao, S.; Tachino, J.; Matsuyama, T.; Kiguchi, T.; Umemura, Y. Effect of fluid administration on scene to traffic collision patients by EMS personnel: A propensity score matched study using population-based ambulance records and nationwide trauma registry in Japan. Eur. J. Trauma Emerg. Surg. 2021, 1-9, (Online ahead of print). [CrossRef]

30. Aoki, M.; Abe, T.; Oshima, K. Association of prehospital epinephrine administration with survival among patients with traumatic cardiac arrest caused by traffic collisions. Sci. Rep. 2019, 9, 9922. [CrossRef] [PubMed]

31. Tsur, A.M.; Nadler, R.; Tsur, N.; Sorkin, A.; Bader, T.; Benov, A.; Glassberg, E.; Chen, J. Prehospital definitive airway is not associated with improved survival in trauma patients. J. Trauma Acute Care Surg. 2020, 89, S237-S241. [CrossRef] [PubMed]

32. Nakahara, S.; Tomio, J.; Takahashi, H.; Ichikawa, M.; Nishida, M.; Morimura, N.; Sakamoto, T. Evaluation of pre-hospital administration of adrenaline (epinephrine) by emergency medical services for patients with out of hospital cardiac arrest in Japan: Controlled propensity matched retro-spective cohort study. BMJ 2013, 347, f6829. [CrossRef] [PubMed]

33. Koike, S.; Tanabe, S.; Ogawa, T.; Akahane, M.; Yasunaga, H.; Horiguchi, H.; Matsumoto, S.; Imamura, T. Effect of time and day of admission on 1-month survival and neurologically favourable 1-month survival in out-of-hospital cardiopulmonary arrest patients. Resuscitation 2011, 82, 863-868. [CrossRef]

34. Wallace, S.K.; Abella, B.S.; Shofer, F.S.; Leary, M.; Agarwal, A.K.; Mechem, C.C.; Gaieski, D.F.; Becker, L.B.; Neumar, R.W.; Band, R.A. Effect of time of day on prehospital care and outcomes after out-of-hospital cardiac arrest. Circulation 2013, 127, 1591-1596. [CrossRef] [PubMed]

35. Peberdy, M.A.; Ornato, J.P.; Larkin, G.L.; Braithwaite, R.S.; Kashner, T.M.; Carey, S.M.; Meaney, P.A.; Cen, L.; Nadkarni, M.V.; Praestgaard, A.H.; et al. National Registry of Cardiopulmonary Resuscitation Investigators. Survival from in-hospital cardiac arrest during nights and weekends. JAMA 2008, 299, 785-792. [CrossRef]

36. Umemura, Y.; Katayama, Y.; Kitamura, T.; Kiyohara, K.; Hirose, T.; Kiguchi, T.; Tachino, J.; Nakao, S.; Nakagawa, Y.; Shimazu, T. Patient age affects sex-based differences in post-traumatic mortality: A national trauma registry study in Japan Eur. J. Trauma Emerg. Surg. 2021, 1-10, (Online ahead of print). [CrossRef]

37. Neumar, R.W.; Nolan, J.P.; Adrie, C.; Aibiki, M.; Berg, R.A.; Böttiger, B.W.; Callaway, C.; Clark, R.S.B.; Geocadin, R.G.; Jauch E.C.; et al. Post-cardiac arrest syndrome: Epidemiology, pathophysiology, treatment, and prognostication. A scientific statement from the International Liaison Committee on Resuscitation; the American Heart Association Emergency Cardiovascular Care Committee; the Council on Cardiovascular Surgery and Anesthesia; the Council on Cardiopulmonary, Perioperative, and Critical Care; the Council on Clinical Cardiology; the Council on Stroke. Circulation 2008, 118, 2452-2458.

38. Yasin, Y.J.; Grivna, M.; Abu-Zidan, F.K. Global impact of COVID-19 pandemic on road traffic collisions. World J. Emerg. Surg. 2021, 16, 51. [CrossRef] 